\title{
Revisão
}

\section{Evolução neuromotora de um recém-nascido pré-termo e a correlação com os fatores perinatais}

\section{Neuromotor evolution of pre-term newly born and correlation with perinatal factors}

Cíntia Salles Brandolis Gregório*; Elizabeth Costa Tang Pinheiro**; Daniela Elaine Oliveira Campos***; Eduardo José Alfaro****

*Fisioterapeuta, especialista em Fisioterapia aplicada a Neonatologia pela UNICAMP, mestranda em Distúrbios do Desenvolvimento pela Universidade Presbiteriana Mackenzie, docente da disciplina de Fisioterapia aplicada a Ginecologia,Obstetricia e Pediatria da Universidade Camilo Castelo Branco-SP, **Fisioterapeuta, mestranda em Educação pela Universidade Brás Cubas, docente da disciplina de Fisioterapia aplicada a Ginecologia,Obstetricia e Pediatria da Universidade Camilo Castelo Branco-SP, FCNM e UniSantana, **Fisioterapeuta, mestranda em Distúrbios do Desenvolvimento pela Universidade Presbiteriana Mackenzie, docente da disciplina de Fisioterapia aplicada a Ginecologia,Obstetrícia e Pediatria da Universidade Camilo Castelo Branco-SP e UniSantana, ****Fisioterapeuta, mestre em Distúrbios do Desenvolvimento pela Universidade Presbiteriana Mackenzie, docente supervisor de estágio em Neuropediatria e Neurologia da Universidade Camilo Castelo Branco-SP e FCNM.

Palavras-chave: prematuro; fatores de risco; evolução neuromotora.

\section{Resumo}

O objetivo deste trabalho é realizar um estudo retrospectivo de uma criança de dois anos de idade, nascida prematuramente e descrever sua evolução neuromotora, assim como os fatores perinatais envolvidos, considerados de risco para distúrbio de desenvolvimento. Mediante revisão de literatura, buscou-se identificar possível correlação dos fatores perinatais envolvidos com a incidência de distúrbios de desenvolvimento. Apesar da evolução neuromotora não apresentar alterações até o presente momento, é necessário acompanhar esta criança, para verificar possível distúrbio de aprendizagem. 
Key-words:

preterm; risk factors; motor evolution.

\begin{abstract}
The goal of this work is to perform a retrospective life-long study of a two years-old child, preterm newborn, in order to describe his motor evolution. The child birth related factors are also considered, since they may introduce risks associated to occurrence of developmental disturbs. These factors were listed, and it has been searched for a possible correlation with the presence of developmental disturbs - such a correlation was searched by means of specialized literature revision. Although the child motor evolution did not suffer changes until current time, a longitudinal tracking study of this child is needed, to verify a possible learning damage.
\end{abstract}

\section{Introdução}

Nos últimos vinte anos, os avanços tecnológicos nos cuidados perinatais e a capacitação dos profissionais envolvidos no acompanhamento de gestações de risco, tem permitido maior sobrevivência de recémnascidos pré-termo (RNPT) com menos de 32 semanas de idade gestacional e/ou peso menor que $1500 \mathrm{~g}$. Esta população de RNPT apresenta o que a literatura tem designado "novas morbidades", uma vez que apresentam alto risco para desvios de desenvolvimento e de seqüelas neuromotoras e sensoriais $[1,2,3]$. As seqüelas mais comuns nesta população são; atraso no desenvol-vimento, distúrbios de aprendizagem, comportamento e paralisia cerebral (P.C.) $[4,5,6,7,8,9]$.

O objetivo deste trabalho é realizar um estudo retrospectivo de uma criança de dois anos de idade, nascida prematuramente e descrever sua evolução neuromotora, assim como os fatores perinatais envolvidos, considerados de risco para distúrbio de desenvolvimento.

A P.C. é um grupo de desordens não progressivas caracterizadas por um distúrbio do tônus e da postura, secundárias a uma disfunção ou malformação do encéfalo em desenvolvimento, que pode ocorrer no período pré, peri ou pós-natal (período que compreende até dois anos após o nascimento). $\mathrm{O}$ quadro clínico pode-se modificar com o passar do tempo, muito embora a P.C. seja uma desordem neurológica fixa $[12,13,14]$. O aumento da incidência de P.C. no início da década de 80 coincidiu com o aumento do índice de sobrevivência dos pequenos prematuros. Uma das possibilidades mais aceitas para este fato, é de que a P.C. 
represente um ponto final de uma cadeia de efeitos aversivos, ocorrendo num período em que o encéfalo está particularmente vulnerável à lesões isquêmicas [15].

Kuban e Leviton [13] relataram em seus estudos, que o índice de P.C. é 25 a 31 vezes mais alto em recém-nascidos com peso de nascimento menor ou igual a $1500 \mathrm{~g}$ (comparado a recém-nascidos de peso normal). Os bebês cujo peso de nascimento é menor que $2500 \mathrm{~g}$ refletem possibilidade de $33 \%$ e apresentar sinais de P.C.. Por outro lado, outros autores relataram que a prematuridade como fator isolado (idade gestacional $<37$ semanas, a contar do $1^{\circ}$ dia da última menstruação [10]), não retarda nem acelera a seqüência do desenvolvimento neuropsicomotor $[16,17]$.

No presente caso,V.S.M., sexo masculino, branco, nasceu de parto vaginal, com 31 semanas de idade gestacional, peso ao nascimento de $770 \mathrm{~g}$ e altura de $33 \mathrm{~cm}$ (RNPT, pequeno para a idade gestacional (PIG), com peso ao nascimento menor do que seria de se esperar, diante de sua idade gestacional [10]), podendo representar alto risco para P.C..

O RNPT PIG também é considerado de risco, pelos efeitos adversos da prematuridade e do crescimento intra-uterino. MacCarton et al em 1996, encontraram maior morbidade cognitiva e neurológica nos RNPT PIG do que nos adequados para a idade gestacional (AIG) - com peso ao nascimento dentro do que seria de se esperar diante de sua idade gestacional [10] -, quando comparados com a idade gestacional. Contudo, na comparação desses recém-nascidos por faixa de peso, não houve diferença estatística entre os grupos [18]. Outro estudo[19] relatou os efeitos da prematuridade e do retardo do crescimento intra-uterino sobre o desenvolvimento motor e cognitivo diferem em seus mecanismos. A habilidade motora foi positivamente associada à idade gestacional e negativa-mente associada ao grau de retardo de crescimento intra-uterino, enquanto a habilidade cognitiva, esteve relacionada ao retardo de crescimento intra-uterino. No presente estudo, além de retardo de crescimento intra-uterino, V.S.M. apresenta a idade gestacional como fator aversivo às habilidades cognitiva e motora, analisadas em tais estudos.
No entanto, outros autores relatam que na ocorrência de retardo de crescimento intrauterino em bebês a termo, corre-se o risco de P.C., mas não em bebês nascidos antes da $33^{a}$ semana de idade gestacional [20,21].

V.S.M. necessitou de ventilação por pressão positiva logo após o nascimento (anóxia perinatal) e foi encaminhado à unidade de terapia intensiva neonatal - sob entubação orotraqueal e ventilação pulmonar assistida ,onde permaneceu por 74 dias (dos quais 21 dias sob ventilação assistida). Neste período V. apresentou algumas intercorrências, consideradas fatores de risco para P.C., como meningite, sepse precoce, displasia broncopulmonar, hemorragia periventricularintraventricular (HPIV) e retinopatia da prematuridade.

A necessidade de ventilação assistida foi positivamente associada à P.C em RNPT, embora não podendo ser utilizada como único fator, pois está intimamente relacionada com a prematuridade e os eventos relacionados à esta, como o baixo peso ao nascimento, a HPIV, crises convulsivas neonatais, displasia broncopulmonar, meningite, sepse e asfixia perinatal, que também são considerados fatores de risco [22].

O tempo de internação do RNPT na unidade de terapia intensiva neonatal parece ter relação nas crianças com diagnóstico de alteração neurológica. Pedromônico et al mostraram em 1998, que os RNPT com tempo de internação superior a 35 dias mostraram evidência de alteração no processo de construção da imagem corporal [23].

A HPIV - sangramento da matriz germinativa subependimária [11] -, é a patologia neurológica mais importante no período neonatal, acometendo principalmente o RNPT com peso ao nascimento menor que $1500 \mathrm{~g}$, ocasionando graves seqüelas motoras e intelectuais, principalmente nos graus III e IV [24]. A presença ou ausência de HPIV é diagnosticada mediante a ultra-sonografia cerebral neonatal. Resultados que apresentem graus lesivos de HPIV (graus III e IV), são considerados de risco para alterações no desenvolvimento [25]. No presente caso, V. apresentou HPIV grau I, portanto não considerado lesivo ou de risco para alterações no desenvolvimento. 
Segundo Tavares et al [26], a idade gestacional ou baixo peso ao nascer $(<1750 \mathrm{~g})$, parto vaginal e sexo masculino são fatores fortemente associados com a presença de HPIV - o que coincide com o presente caso.

Mello et al [27] realizaram um estudo preditivo da presença de HPIV, em relação ao desenvolvimento motor e cognitivo de prematuros de muito baixo peso, após 12 meses de idade corrigida. Concluíram que diante da ausência de HPIV, a probabilidade de uma criança ter desenvolvimento motor e cognitivo normais é de $85 \%$. Ou seja, prematuros de muito baixo peso, com ausência de HPIV (ou graus não lesivos de HPIV), como no presente estudo, apresentam probabilidade estatística de $15 \%$ de acontecer distúrbio no desenvolvimento.

A displasia broncopulmonar é uma patologia crônica, característica de recémnascidos ventilados em pressão positiva e/ou expostos a altos níveis de oxigênio inspirado. Estas crianças requerem maior tempo de internação por dependência crônica de oxigênio. V., durante o período de internação, permaneceu 21 dias sob ventilação assistida e após a alta hospitalar permaneceu sob oxigênioterapia domiciliar durante 4 meses período em que realizou acompanhamento fisioterapêutico domiciliar diário (4 vezes ao dia). Alguns estudos mostram que a displasia broncopulmonar pode estar relacionada com distúrbios no desenvolvimento.

Northway em 1979, estudou a incidência de alterações do desenvolvimento neuropsicomotor em crianças com displasia broncopulmonar, encontrando $34 \%$ com seqüelas graves, incluindo outros itens, tais como: paralisia cerebral, retardo mental, déficit visual e déficit auditivo [28].

Sauve e Singhal em 1985 estudaram 179 recém-nascidos com displasia broncopulmonar e 112 controles pareados por peso e ano de nascimento. O grupo de recémnascidos com displasia broncopulmonar apresentou pequeno aumento de incidência de alterações no desenvolvimento neuromotor e déficit auditivo, porém este resultado não foi significativo [29]. Meisels em 1986, também mostrou em seu estudo que o desenvolvimento neuropsicomotor dos pacientes com displasia broncopulmonar é, em geral, mais afetado do que em recém-nascidos controle [32].

Sweeney \& Swanson em 1998, relataram que bebês de baixo peso ao nascimento geralmente exibem instabilidade neurológica, devido ao seu estado clínico, e não à disfunção do sistema nervoso central. Uma das condições que leva a esta condição é a displasia broncopulmonar. Os bebês com doença pulmonar crônica exibem baixo tono muscular, atraso nas funções motoras e reações de equilíbrio imaturas [38].

Outros autores sugerem, que o desenvolvimento neuropsicomotor está relacionado a eventos perinatais e neonatais, e não à presença ou ausência de displasia broncopulmonar. Assim, não se pode relacionar a displasia broncopulmonar como fator isolado associada às seqüelas motoras [30,31].

A asfixia perinatal não tem sido relacionada às seqüelas neuromotoras tardias e a paralisia cerebral $[33,12,34]$. Porém, pode-se produzir alterações transitórias, resultando em uma encefalopatia manifesta, como hipotonia proximal dos membros inferiores de RNPT, convulsões e tremores, 72 horas após o nascimento [35]. Drillien et al [4], avaliaram o desenvolvimento neuropsicomotor de 281 recém-nascidos com peso ao nascimento inferior a $2000 \mathrm{~g}$, e constataram que $40 \%$ destas crianças apresentaram alterações neuromotoras transitórias nos primeiros meses de vida. Estes autores denominaram este fenômeno de distonia transitória associada ao baixo peso ao nascer, que se traduz clinicamente de hipotonia proximal em membros inferiores, hipertonia extensora do pescoço, irritabilidade e letargia.

Muitas vezes são avaliados fatores não específicos para quantificar a asfixia perinatal. O boletim de Apgar, que é utilizado rotineiramente nos países ocidentais para a avaliação das condições clínicas do recémnascido no momento do parto, não apresenta boa correlação com a asfixia perinatal em recém-nascidos com idade gestacional inferior a 32 semanas [36]. Isto explicaria o fato de V. apresentar asfixia perinatal e um boletim de Apgar considerado contraditório para tal evento ( $1^{\circ}$ minuto, 8 e $5^{\circ}$ minuto 9 ). 
Todos os fenômenos descritos podem ocorrer concomitantemente, influindo negativamente no prognóstico neuropsicomotor.

Os estudos na literatura têm demonstrado a importância do caráter precoce da intervenção. Os primeiros dois anos de vida constituem o período crítico para a estimulação, devido à plasticidade cerebral e ao rápido período de crescimento e desenvolvimento. Em 1987, Resnick et al demonstraram redução significativa na prevalência de atraso no desenvolvimento neuropsicomotor em 124 crianças, submetidas a um programa de intervenção precoce desde o período de internação até o final do segundo ano de vida, quando comparado a um grupo controle não submetido à intervenção [39]. Outros estudos demonstraram melhora no crescimento somático e na função motora após estimulação visual, auditiva, tátil,cinestésica e vestibular no período neonatal $[40,41,42]$.

No presente estudo, V. realizou durante o período de internação, acompanhamento fisioterapêutico (estimulação motora e fisioterapia respiratória). No período pré-alta os pais receberam orientações quanto a importância da continuidade na intervenção terapêutica (estimulação). Durante os primeiros quatro meses pós- alta, V. recebeu acompanhamento fisioterapêutico diário e, em seguida, iniciou fisioterapia na clínica-escola da Universidade Camilo Castelo Branco estando com 4

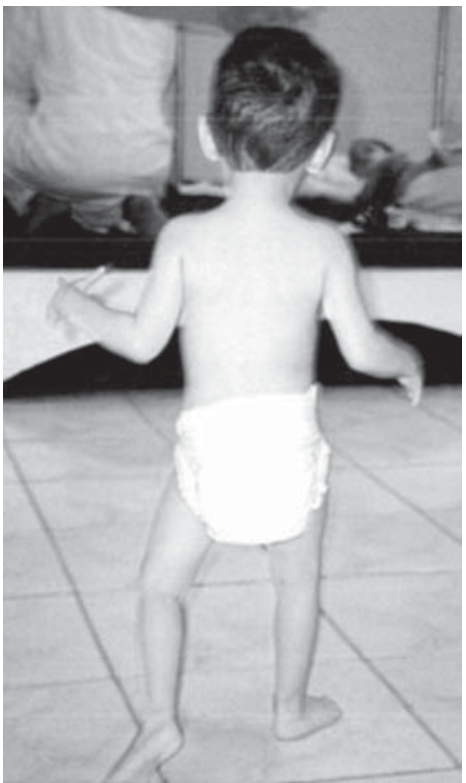

rapêuitco foi intensivo (5 vezes semanais). A família foi orientada quanto a p o s i c i o $\mathrm{n}$ amentos corretos e exercícios para serem realizadas em casa. V., durante 1 ano e 4 meses, não apresentou intercorrências respiratórias.

Durante sua evolução neuromotora, V. adquiriu todas as etapas posturais. Com 1 ano de 3 meses de idade cronológica deambulava com apoio e 3 meses depois, deambulava independentemente.

Atualmente, com dois anos de idade cronológica, apresenta retração de cintura escapular, que evidencia-se em todas as posturas, principalmente durante o correr (fotos). A função bimanual e as pinças estão presentes e sem alterações.

\section{Comentários}

A retração de cintura escapular deve-se, provavelmente, à permanência em decúbito dorsal de 74 dias durante o período de internação. Talvez por fatores fisiológicos, já que o RNPT encontra-se em condições bem diferentes do ambiente intra-uterino. Este, além de fornecer suporte fisiológico e temperatura adequada, priva o feto da ação da gravidade - pois sua musculatura esquelética ainda não atingiu maturação suficiente para vencê-la [46]. Assim, pode-se supor que a longa permanência desta musculatura esquelética imatura sob a ação da gravidade, seja responsável por esta retração de cintura escapular.

Mediante revisão de literatura, pode-se dizer que a intervenção fisioterapêutica precoce é favorável em casos de RNPT PIG. 
Mesmo na presença de fatores perinatais aversivos ao desenvolvimento neuropsicomotor normal, a intervenção precoce mostrou ser favorável quanto ao desenvolvimento neuromotor. No entanto, estas crianças devem ser acompanhadas até a idade escolar, já que muitos estudos mostram que grande porcentagem apresentam déficit de aprendizagem.

O estudo de Drillien et al. [4], mostrou que $14 \%$ dos recém-nascidos de peso abaixo de $1500 \mathrm{~g}$ apresentavam algum tipo de déficit sensorial, mental ou neurológico, durante o primeiro ano de vida. Aos seis anos de idade, $92 \%$ das crianças deste estudo freqüentavam escola normal, sendo que $18 \%$ delas apresentavam algum déficit de aprendizagem.

Calame et al. ressaltaram a importância da intervenção precoce do RNPT de muito baixo peso. E relataram que, embora os casos de paralisia cerebral tivessem diminuído, as incidências de distúrbios leves e de dificuldades eram menos avaliadas [43]. Esse estudo mostra a relação entre pequenas anormalidades neurológicas no primeiro ano de vida e fracasso escolar, observado em cerca de $23 \%$ das crianças. Em $12 \%$ delas foi constatado déficit sensório motor grave, e houve alta incidência de desvios de comportamento, como hiperatividade, ansiedade e irritabilidade, com comprometimento da escolaridade. Outros autores também observaram que as alterações neuromotoras no primeiro ano de vida, mesmo se transitórias, em recém -nascidos pré-termo submetidos a fatores de risco perinatais, estão associadas a mau desempenho escolar $[4,44,45]$.

Desse modo, torna-se necessário um acompanhamento longitudinal dessa criança, para observar o possível acometimento na aprendizagem psicomotora. Até o presente momento, não evidencia-se alterações de ordem neuromotoras.

\section{Referências}

1. Vergara E. Foudations for practice in the neonatal intensive care unit and early intervention. Rockville: AOTA Inc., $1993 ; 335$.

2. Goyen TA, Lui K, Woods T. Visual-motor, visual-perceptual and fine motor outcomes in very-low- birthweight children at 5 years. Dev Med Child Neurol 1998;40:70-81.

3. Luoma L, Herrgard E, Martikainen A. Neuropsychological analysis of the visuomotor problems in children born preterm at $<32$ weeks of gestacion: a 5year prospective follow-up. Dev Med Child Neurol 1998; 40:21-30.

4. Drillien CM, Thomsom JM,Burgoyne K. Low birthweight children in early school age: a longitudinal study. Dev Med Child Neurol 1980;22:26-47.

5. Msall ME, Buck GM, Rogers BT et al. Risk factors for major neurodevelopmental impairments and need for special education resources in extremely premature infants. J Pediatr 1991;119 (4): 606-14.

6. Barbosa NMM, Cabral AHL, Silva OPV et al. "Follow-up" do bebê de risco. Temas Pediatr 1993;(53):1-36.

7. Coello FN, Gontijo APB, Figueiredo EM et al. O que os pais de recém-nascidos de alto risco conhecem sobre desenvolvimento infantil? Temas Desenv 1998; 7(38): 32-38.

8. Gosch A, Brambring M, Gennat $\mathrm{H}$ et al. Longitudinal study of neuropsychological outcome in blind extremely-lowbirthweight children. . Dev Med Child Neurol 1997; 39: 297-304.

9. Campbell SK.The infant at risk for developmental disability. In: Campbell SK,ed. Decision making in pediatric physical therapy. New York: Churchill Livingstone 1999; 260-332.

10. Kelnar, C.J.; Harley,D.(1995). O recémnascido doente - diagnóstico e tratamento em neonatologia. $3^{a}$ ed. São Paulo: Santos.

11. Volpe,J.J. Intraventricular hemorrage in the premature infant: morphologic characteristics. Fetal and Neonatal physiology. Phyladelphia,W.B. Saunders Company,1992; 1598-1608.

12. Nelson KB, Swaiman KF, Russman BS. (1994). Cerebral Palsy. Pediatric Neurology - Principles and Praitice. (I), 471-488.

13. Kuban KCK, Leviton A. Cerebral Palsy. The New England Journal of Medicine 1994; (20): 188-195.

14. Piovesana AMS. Paralisia Cerebral: 
Contribuição de estudo por imagem. Paralisia Cerebral : Aspectos Práticos. (I), 9-37, 1998.

15. Murphy D, Sellers S, Mackenzie IZ et al. Case control study of antenatal and intrapartum risk factors for cerebral palsy in very preterm singleton babies. Lancet 1995; 346: 1449-54.

16. Gesell A, Amatruda CS. et al. Effect of gestacional age on neurological functioning of the very low-birthweight infant at 40 weeks. Dev. Med.Child.Neurol. 1985; 27: 596-605.

17. Saint-Anne Dargassies, S. La maturacion neurologique des prématurés. Éstudes Néonatales 1955; 4:71-116

18. MacCarton, c.m.; Wallace, I.F. et al. Cognitive and neurologic development of the premature, small for gestacional age infant through age 6 : comparison by birth weight and gestacional age. Pediatrics 1996; 98:1167-78.

19. Huntton JL, Pharoah PO et al. Differential effects of preterm birth and small gestacional age on cognitive and motor development. Arch Dis Child 1997;76:75-81.

20. Gaffney G, Sellers S et al. Case control study of intrapartum care, cerebral palsy and perinatal death. BMJ 1994;308:743-50.

21. Murphy D, Sellers S et al. Case control study of antenatal and intrapartum risk factors for cerebral palsy in very preterm singleton babies. Lancet 1995;346:1449-54.

22. Graziani LJ, Spitzer AR et al. Mechanical ventilation in preterm infants neurosonographic and developmental studies. Pediatrics 1992;90:515-22.

23. Pedromônico RM, Azevedo MF, Kopelmam BI. Recém-nascidos pré-termo internados em unidades de terapia intensiva: desenvolvimento da conduta interativa no primeiro ano de vida. $J$ Pediat 1998;74 (4):284-90.

24. Volpe JJ. Intraventricular hemorrage: periventricular-intraventricular hemorrhage of the premature infant. Neurology of the newborn. 2ed, Phyladelphia,W.B. Saunders Company,1987; 311-61.

25. Behnke M, Eyler FD et al. Cranial ultrasound abnormalities identified at birth: their relationship to perinatal risk and neurobehavioral outcome. Pediatrics 1999;103(4): 41.

26. Tavares EC, Corrêa FF, Viana MB. Fatores de risco para hemorragias periintraventriculares em recém-nascidos com peso menor de 2000 gramas. J Pediat 1998;74(1):17-23.

27. Mello RR Meio BB et al. Ultra-sonografia cerebral neonatal normal no prematuro é possível tranqüilizar os pais? J Pediat 1999;75(1):45-49.

28. Northway WH. Observations on broncopulmonary dysplasia. J Pediat 1979; 95: 815-17.

29. Sauve RS, Singhal N. Long-term morbidity of infants with broncopulmonary dysplasia. Pediatrics, 1985;6:725-33.

30. MarkestadT, fithardinge PM. Growth and development in children recovering from broncopulmonary dysplasia. J Pediat 1981; 98:597-602.

31. Gray PH, BurnsYR et al. Neurodevelopmental outcome of preterm infants with broncopulmonary dysplasia. Arch Dis Child1995;73:128-34.

32. Meisels SJ, Plunkett JW et al. Growth and development of preterm infants with respiratory syndrome and bronchopulmonary dysplasia. Pediatrics 1986;77:345-52.

33. Blair E, Stanley FJ. Intrapartum asphyxia: a rare cause of cerebral palsy. J Pediat 1988; 112:515-9.

34. Naeye RL, Peters EC et al. Origins of cerebral palsy. AJDC 1989;143:1154-61.

35. Drillien CM. Abnormal neurological signs in the first year of life in low birthweight infants:possible prognostic significance. Dev Med Child Neurol 1972; 14: 705-11.

36. Catilin EA. Carpenter NW et al. A simplified method for diagnosis of gestacional age in the newborn infant. $J$ Pediatr 1986;109: 865-8

37. Legido A, Clancy RR, Berman PH. Neurologic outcome after eletroencephalographically proven neonatal seizures. Pediatrics 1991; 88:583-96.

38. Resnick MB, Eyler FD et al. Developmental intervention for low birth weight infants: improved early 
developmental outcome. Pediatrics 1987; 80:68-74.

39. Resnick MB, Eyler FD et al. Developmental intervention for low birth weight infants: improved early developmental outcome. Pediatrics 1987; 80:68-74.

40. Kraumer LI, Pierpont ME. Rocking waterbeds and auditory stimili to enhance growth of preterm infants. J Pediatr 1976; 88:297-9.

41. Leib SA, Benfield G, Guidubaldi J. Effects of early intervention and stimulation on the preterm infant, Pediatrics 1980;66:8390.

42. Field TM, Schanberg SM, Scafidi F et al. Tactile/Kinesthetic stimulation effects on preterm neonates. Pediatrics 1986; 77:654-8.
43. Calame A, Fawer CL et al. Neurodevelopmental outcome and school performance of very-low-birth-weith infants at 8 years of age. Eur $J$ Pediatr 1986;145 (6): 461-6.

44. Amiel-Tison C.; Dubê R et al. Outcome at age five years of full-term infants with transient neurologic abnormalities in the first year of life. In: Stern L., BardiisHansen B. Intensive care in the newborn. New York: Masson 1983; 247-58.

45. Amiel-Tison C, Dubê R. Signification des anomalies neuro-motrices transitoires; correlations avec les difficultés de láge scolaire. Ann Pediatr 1985; 32 (1): 55-61.

46. Dubowitz V. Enzyme histochemistry of developing human muscle. Nature 1966; 211: 884-885.

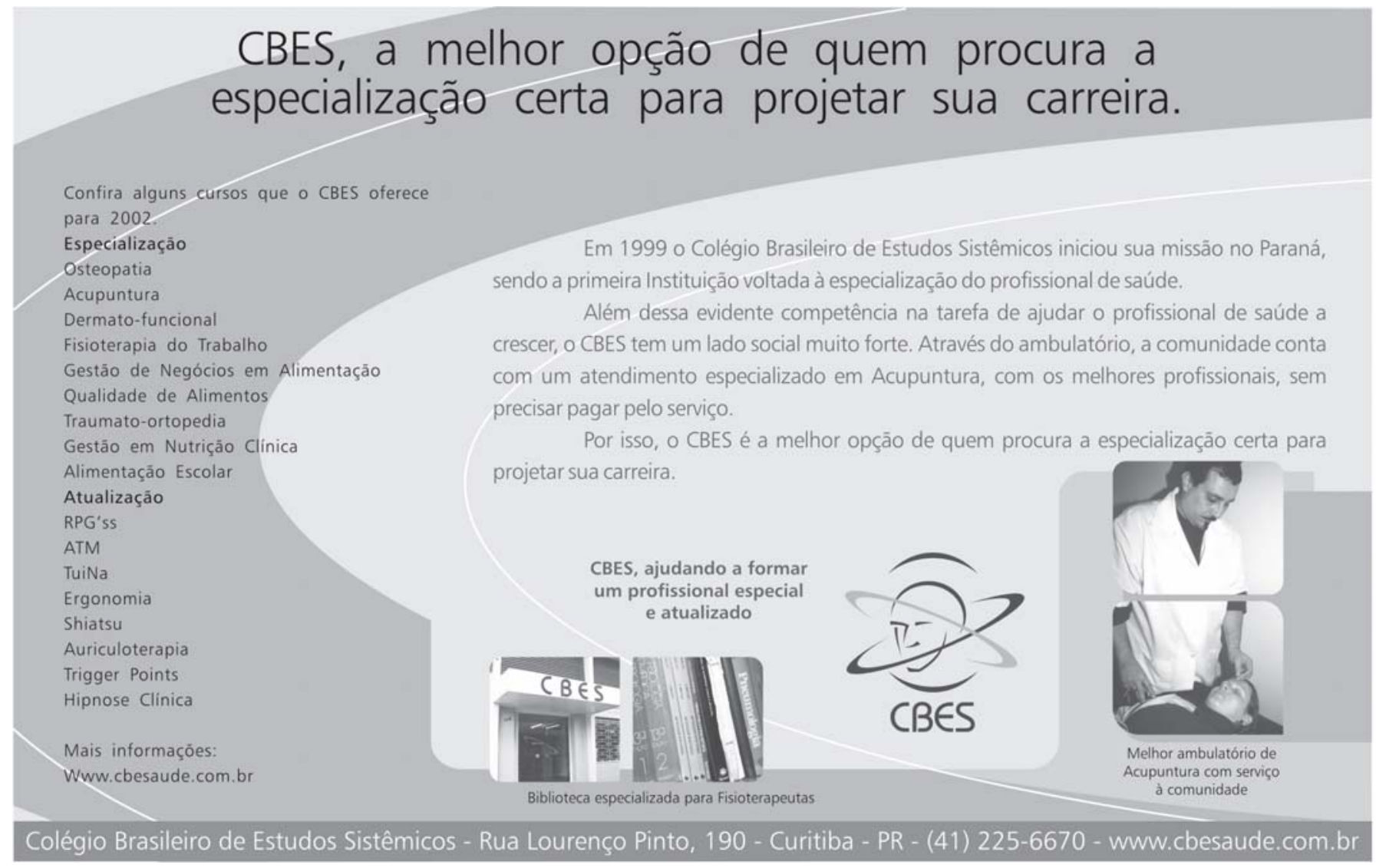

